\title{
Wider die Binarität - Psychoanalyse und Queer Theory
}

\author{
Esther Hutfless (Wien)
}

Zusammenfassung: Wie Michel Foucault in seiner Analyse Sexualität und Wahrheit I gezeigt hat, ist die Kategorie «Homosexualität» Mitte bis Ende des 19. Jahrhunderts als diskursive Konstruktion entstanden. "Homosexualität» als Kategorie entwickelte sich also nicht als gelebte Identität, sondern zunächst als Markierungskategorie für deviante Subjekte.

Die Psychoanalyse hat von Beginn an versucht, die Entgegensetzung von Abweichung und Norm aufzulösen, indem sie Homosexuelles im Heterosexuellen und die Perversion in jeder Sexualität verortet hat. Zugleich jedoch hat die Psychoanalyse diese Dichotomisierung mit einer Pathologisierung der Homosexualität auch fortgeschrieben. Konzepte innerhalb der Psychoanalyse, die die Unterscheidung von Homo- und Heterosexualität in Frage stellen, werden auch heute kaum aufgegriffen, während nach wie vor nach den Ursachen vor allem ersterer gefahndet wird.

In meinem Beitrag möchte ich die Kritik an der binären Entgegensetzung von Homo- und Heterosexualität und die Entwicklung queerer Ansätze nachzeichnen und die Queer Theory mit der Psychoanalyse in einen produktiven Dialog bringen. Ansätze der Queer Theory können für eine nicht-pathologisierende Auseinandersetzung mit Homo-, aber auch Transsexualität in der Psychoanalyse wichtige Impulse liefern.

Schlüsselwörter: Queer Theory, Psychoanalyse, Transgender, Homosexualität

Die Psychoanalyse war und ist, ebenso wie die abendländische Denktradition, geprägt vom Denken in binären und dichotomen Strukturen, die sich in eine hierarchische Ordnung eingebettet finden. In der Psychoanalyse werden diese hierarchischen Strukturen auch an entwicklungspsychologische gebunden (vgl. Drescher, 2007, S. 219). Heterosexualität und Homosexualität stellen damit nicht bloss ein Gegensatzpaar dar; Heterosexualität wird als die reifere Entwicklungsform der Homosexualität hierarchisch übergeordnet.

Sigmund Freud jedoch hat seit den Anfängen seiner psychoanalytischen Forschung unter anderem versucht, diese Entgegensetzungen aufzulösen, indem er 
Homosexuelles auch in der Heterosexualität (Freud, 1905, S. 44) und die Perversion in jeder Sexualität verortet hat. Mit diesem radikal dekonstruktiven Denken, das sowohl die Dichotomie von Homo- und Heterosexualität in Frage stellt als auch das Perverse aus einem pathologisierenden Diskurs herauslöst - indem jegliche Sexualität als losgelöst von der Fortpflanzungsfunktion, durchsetzt von unbewusstem Triebgeschehen, verstanden wird -, legt Freud gewissermassen schon den Grundstein für ein «queeres» Verständnis von Sexualität (Dean \& Lane, 2001, S. 5). Freud selbst hält an dieser Position jedoch nicht konsequent fest und in der weiteren theoretischen Entwicklung der Psychoanalyse wurde die Dichotomisierung und Hierarchisierung, die mit der Pathologisierung von Homosexualität einhergeht, ebenso weiter fortgeschrieben. Konzepte innerhalb der Psychoanalyse, die die Entgegensetzung von Hetero- und Homosexualität in Frage stellen und die Pathologisierung letzterer kritisch hinterfragen und dekonstruieren, werden zumindest vom breiten psychoanalytischen Mainstream kaum aufgegriffen.

Ich möchte daher im Folgenden queer-theoretische Ansätze, die die Kritik und Dekonstruktion eindeutiger Identitätskategorien ins Zentrum ihrer Auseinandersetzung stellen, in einen produktiven Dialog mit der Psychoanalyse bringen. Dies kann insbesondere auch der grossteils noch immer durch heteronormative Positionen geprägten psychoanalytischen Auseinandersetzung mit Homo- und Heterosexualität neue Impulse liefern.

\section{Von Gay and Lesbian Studies zu Queer Theory}

In den 70er Jahren haben die sich neu entwickelnden Gay and Lesbian Studies begonnen, Homosexualität als historische und soziale Kategorie zu untersuchen: So wurde nicht mehr Homosexualität als Problem betrachtet, sondern der gesellschaftliche Umgang damit, und es wurde genealogisch gezeigt, dass Homosexualität als sexuelle Identität ein gesellschaftlich hervorgebrachtes Konstrukt darstellt, das nicht zu allen Zeiten an allen Orten existierte. Zur gleichen Zeit wurde Homosexualität aber für einige auch zur politisch bedeutenden Identität - Feminist*innen ${ }^{1}$ haben etwa dafür argumentiert, LesbischSein als widerständige Praxis gegen das Patriarchat und gegen heterosexuelle Familiennormen zu verstehen.

Die Binarität von Homo- und Heterosexualität wurde trotz dekonstruktivistischer Ansätze fortgeschrieben und es wurde zunächst nicht grundsätzlich danach gefragt, warum Menschen an sexuellen Kategorien gemessen werden, wie Sexualität überhaupt zu einer derart zentralen Identitätskategorie werden konnte und warum Heterosexualität, die man als ebenso historisch hervorgebracht verste- 
hen muss, sich als normale Ausrichtung behaupten konnte (Richardson \& Seidman, 2002, S. 2). Um die politischen Ziele der Gleichstellung und Antidiskriminierung zu erlangen, hat die «Mainstream»-Schwulen- und Lesben-Bewegung nicht nur Homosexualität als politische Identität begriffen, sondern auch dafür argumentiert, Homosexualität als angeboren zu verstehen: Homosexualität stelle also eine Naturgegebenheit dar - man könne von ihr somit weder geheilt werden noch liege ihr eine persönliche Entscheidung zu Grunde. Geht es grossen Teilen der Lesben- und Schwulenbewegung nach wie vor eher um Gleichstellung verbunden mit «Normalisierungsprozessen», hinterfragen queere Theorien sowohl die Idee einer gemeinsamen homosexuellen Identität als auch jegliche Vorstellung des «Normalen». Queer-Theoretiker*innen denken Sexualität, sexuelle Orientierung und geschlechtliche Identität als dynamisch, instabil und prozesshaft, zugleich jedoch auch stets eingebunden in diskursive Konstruktionsformen: "Thus one tension that queer theory throws into relief is that between lesbians and gays who want a respectable, normal existence, and those who want to use their sexuality politically to disrupt normality, with its gender hierarchies, racial discrimination, and conventional expectations about 'family life' for all” (Dean \& Lane, 2001, S. 21). Queer Theory entstand aus einer kritischen Bewegung jenen Identitätskonzepten gegenüber, wie sie sich auch in der Lesben- und Schwulenbewegung verfestigt hatten. Dieser wurde unter anderem von Bisexuellen, Transgenders, People* of Color und Frauen* vorgeworfen, dass sie nicht nur weiss und männlich geprägt sei, sondern selbst wiederum alternative Geschlechtskonzeptionen, Sexualitäten und Identitäten ausschliesse (Richardson \& Seidman, 2002, S. 3). Queer-theoretische Ansätze argumentieren daher nicht für politischen Widerstand, der sich auf individuelle «ausgegrenzte» Identitäten stützt, sondern für eine Politik, die sich allen fixierenden Identitäten und Normierungen widersetzt. Aus diesem Grund nehmen queere Ansätze häufig im Sinne der Intersektionalität auch andere Unterdrückungsformen, wie etwa «Race», «Class», «Color», «Disability» usw. in den Blick (Dean \& Lane, 2001, S. 7).

Es ist insbesondere das kritische und dekonstruktive Potenzial der Queer Theory ${ }^{2}$, das es ermöglicht, all jene - auch impliziten - Vorannahmen, die den traditionellen binären Kategorien von männlich/weiblich, homosexuell/heterosexuell usw. zu Grunde liegen, und zudem auch die Art und Weise, wie Identitäten und Subjekte entstehen - nämlich durch sozial, kulturell, medizinisch, sprachlich usw. konstruierte Normen, Vorstellungen und Prozesse -, zu entlarven und zu hinterfragen. Das Konzept «queer» stellt daher den Versuch dar, Sexualitäten und Geschlechter jenseits von fixen Identitätskategorien zu denken. Dieser Ansatz resul- 
tiert aus der Erkenntnis, dass das Identitätsdenken selbst wesentlicher Bestandteil jenes Prozesses ist, der Ausschlüsse, Abwertungen und Pathologisierungen produziert. Sowohl ausgehend von Michel Foucaults Untersuchungen in Sexualität und Wahrheit (Foucault, 1983) als auch ausgehend von Judith Butlers feministischer Auseinandersetzung etwa im Unbehagen der Geschlechter (Butler, 1991) erweisen sich Identitätskategorien wie «homosexuell», «schwul», «lesbisch» usw. selbst als normalisierend. Foucault zeigt, dass die Bildung derartiger Kategorien ideengeschichtlich eine sehr junge Entwicklung darstellt und daher die im 20. Jahrhundert weit verbreitete Idee, im sexuellen Begehren würde sich die Wahrheit des Subjekts offenbaren, eine historische Konstruktion sei, die das Subjekt letztlich einer diskursiven und disziplinierenden Macht unterstelle. Homosexualität hat, wie Tim Dean und Christopher Lane betonen, also eine Geschichte und die Psychoanalyse hat einen durchaus komplexen Teil dieser Geschichte mitgeprägt (Dean \& Lane, 2001, S. 8). Denn während in psychoanalytischen Diskursen einerseits mitVerweis auf das homosexuelle Begehren Urteile über die reife oder unreife oder über die innerste Problematik eines Subjekts getroffen werden, hat Freud andererseits die Homosexualität als allgemeines Potenzial jeder menschlichen Sexualität verstanden.

\section{Queer Theory und Psychoanalyse}

Das Verhältnis von Schwulen, Lesben, Trans* und Gender Queers zur Psychoanalyse war und ist noch immer ein gespaltenes. Wie Tim Dean und Christopher Lane anmerken, haben LGBTIQ* ${ }^{3}$ die negativen Wirkungen psychoanalytischer Institutionen - ihren normalisierenden Moralismus, ihre disziplinierende Praxis - viel mehr zu spüren bekommen, als dass ihnen mit nichtheterosexistischen, wertschätzenden Haltungen begegnet worden wäre (Dean \& Lane, 2001, S. 5 f.). Trotz aller Kritik an der Psychoanalyse gibt es jedoch vereinzelt Theoretiker*innen aus dem Bereich der Gay and Lesbian Studies und der Queer Theory, die sich insbesondere mit der Psychoanalyse auseinandergesetzt haben und hier zunächst «von ausserhalb» wegweisende Forschungsarbeit geleistet haben, u. a. sind dies Leo Bersani, Guy Hocquenghem, Teresa de Lauretis, Tim Dean und Judith Butler.

Beeinflusst durch Michel Foucault und Gilles Deleuze hat Hocquenghem Mitte der 70er Jahre psychoanalytische Konzepte aufgegriffen und ausgehend davon sowohl das Konzept des Ödipuskomplexes samt der «Ödipalisierung der Homosexualität» als auch das Konzept der Kernfamilie einer Kritik unterzogen. Er entlarvt sowohl die Zweigeschlechtlichkeit als auch die Unterscheidung zwischen 
Homo- und Heterosexualität als willkürlich, da beide Binär-Figuren jeweils durch die Machtwirkung des familiär-ödipalen Systems strukturiert und normalisiert werden (Hocquenghem, 1974). Sowohl in Das Unbehagen der Geschlechter als auch in Körper von Gewicht und Psyche der Macht arbeitet die Philosoph*in Judith Butler - die als eine der Begründer*innen der Queer Theory gilt und stark durch das Werk Foucaults beeinflusst ist - mit psychoanalytischen Ansätzen. Im Unbehagen beschäftigt sich Butler kritisch mit den Ansätzen Jacques Lacans und Joan Rivieres zur Homosexualität. Sie macht Lacans Grundverständnis der symbolischen Ordnung als Instanz, der sich das Subjekt notwendigerweise unterwerfen muss, für ihre Konzeption einer subjekt- und damit auch geschlechtsidentitätsstiftenden Machtwirkung produktiv. Die psychische Annahme des Geschlechts versteht Butler schliesslich ausgehend von Freuds Melancholie-Konzept, indem sie den Verlust eines Liebesobjektes als bedeutsam für die Annahme einer Geschlechtsidentität wertet (Butler, 1991). In Psyche der Macht führt Butler diese Überlegungen fort. Sie schreibt, dass die Heterosexualität nicht erst durch das Inzestverbot zustande käme, sondern diesem bereits ein grundlegenderes Homosexualitätsverbot zugrunde läge, das bereits vor dem Auftreten ödipaler Konflikte ausgebildet sein müsse. Dieses grundlegendeVerbot führe dazu, dass derVerlust des homosexuellen Liebesobjekts nicht betrauert werden könne und zu einer melancholischen Inkorporation führe. «Die Identifizierung enthält also in sich sowohl das Verbot wie das Begehren und verleibt sich somit den unbetrauerten Verlust der homosexuellen Besetzung ein. Wenn man nur in dem Masse Mädchen ist, in dem man kein Mädchen will, dann wird das Verlangen nach einem Mädchen das Mädchensein in Frage stellen [...]» (Butler, 2001, S. 129). Männlichkeit und Weiblichkeit entstehen mit Butler also durch Identifizierungen, die mit einem nicht betrauerbaren Verlust einhergehen. In Körper von Gewicht greift Butler nochmals anders auf psychoanalytische Konzepte - vor allem jene Jacques Lacans - zurück. Hier entwickelt Butler ausgehend von einer dekonstruktiven Auseinandersetzung mit Lacans Konzept des Phallus eine kritische Neuformulierung eines «morphologischen Imaginären», indem sie die Figur des lesbischen Phallus einsetzt. Mit dem Konzept des lesbischen Phallus geht es Butler darum, «einen diskursiven Ort zu eröffnen, an dem die stillschweigenden politischen Beziehungen überprüft werden, die die Aufteilung zwischen Köperzonen und Körperganzem, zwischen der Anatomie und dem Imaginären, der Körperlichkeit und der Psyche konstituieren und in diesen Aufteilungen Bestand haben» (Butler, 1997, S. 111).

Leo Bersani und Teresa de Lauretis arbeiten affirmierend mit psychoanalytischen Konzepten, vor allem mit jenen Jean Laplanches. In The Practice of 
Love. Lesbian Sexuality and Perverse Desire konzeptionalisiert de Lauretis ausgehend von Freud und Laplanche einen Ansatz, lesbisches Begehren jenseits einer heterosexuellen Matrix zu verstehen. Als Bezugspunkt für weibliches/lesbisches Begehren rückt de Lauretis den phantasmatischen weiblichen Körper ins Zentrum, der durch die Kastration beständig in seiner Existenz bedroht wird. Der Signifikant des Begehrens ist nach de Lauretis daher nicht der Phallus, sondern der Fetisch, der den weiblichen Körper selbst adressiert: "In my reading of perverse desire, however, the mediating term, the signifier of desire, is not the paternal phallus but the fetish. Produced in the disavowal of castration, the fetish retains the active - phallic and genital - valences acquired by the drives in the subject's passage through the complete Oedipus complex (negative and positive) and the apprehension of sexual difference; but fetishistic or perverse desire goes beyond the Oedipus complex and in its own way resolves it" (de Lauretis, 1994, S. 289).

Während de Lauretis das Ausgehen vom Mangel in psychoanalytischen Theorien der Subjektgenese grundsätzlich anerkennt, versucht Leo Bersani Begehren jenseits von Mangelkonzeptionen zu verstehen ${ }^{4}$ (Bersani, 1987, 1995). In Homos entwirft Bersani «homo-ness» als «anti-identitäre Identität» (1995, S. 101). Im Unterschied zu queeren Ansätzen hält er es politisch für wichtig, an einer homosexuellen Identität im weitesten Sinne festzuhalten, denn die Auslöschung einer solchen sei genau das, was homophobe Bewegungen anstreben. Er hinterfragt das Konzept der "sexuellen Identität», da sich Sexualität und Identität seinem Verständnis nach grundlegend ausschliessen und entwickelt ausgehend von Laplanches begrifflichem Konzept des «ébrandlement» eine Vorstellung menschlicher Sexualität, in der das schwache Ich von lustvollen bis schmerzhaften Erregungen überflutet wird. Dieses Verständnis von Sexualität findet sich paradigmatisch gerade nicht im reproduktiven heterosexuellen Paar, sondern stellt eine Qualität von homo-ness dar, die sich aber nicht auf Homosexualität beschränken lässt. Bersani wendet hier nicht nur rechte, homophobe Diskurse über die Destruktivität von Homosexualität ins Positive, sondern greift in weiterer Folge auch das jouissance-Konzept von Lacan produktiv auf: "This, in any case, is what Freud appears to be moving toward as a definition of the sexual: an aptitude for the defeat of power by pleasure, the human subject's potential for a jouissance in which the subject is momentarily undone" (Bersani, 1995, S. 100). Damit richtet sich Bersani nicht nur gegen Mangelkonzeptionen in der Psychoanalyse, sondern auch gegen das Verständnis des homosexuellen Subjekts als narzisstisches. Tim Dean entwickelt in Beyond Sexuality mit und ausgehend von Lacans psychoanalytischen Ansätzen ein queeres Verständnis von Sexualität und Subjektivität. Er 
arbeitet insbesondere mit Lacans Konzept des Realen, das seiner Auffassung nach als sich entziehendes, traumatisches, subversives Element die Heteronormativität in ihren symbolischen und imaginären Ordnungen in Frage stellt und das er in einigen Aspekten mit Freuds Auffassung des Unbewussten in Zusammenhang bringt. Seiner Auffassung nach kann die Queer Theory hier viel aus der Psychoanalyse gewinnen: "queer theory has something to gain from psychoanalytic alignment of sex with the unconscious, since this makes sexuality refractive, maladaptive, and therefore always to some extent perverse. In this respect, queer theory is right to claim that perverse sexuality is inherently subversive” (Dean, 2000, S. 245).

Ausgehend von diesen kritischen Auseinandersetzungen mit der Psychoanalyse haben sich schliesslich auch innerhalb der Psychoanalyse vereinzelt produktive Felder entwickelt, die sich an der Schnittstelle von Psychoanalyse und Gender Studies bzw. Queer Theory bewegen und die psychoanalytische Theorien in diesem Feld neu verorten. Die 90er Jahre in den USA stellen hier in zweifacher Hinsicht eine bedeutsame Dekade dar: Einerseits haben sich zu jener Zeit die QueerTheorien aus dekonstruktiven Ansätzen der Gay and Lesbian- bzw. Gender Studies und der Feministischen Theorien entwickelt, andererseits stellen die 90er Jahre jene Periode dar, in der sich die ersten Analytiker*innen (sie hatten ihre Ausbildung noch zu einer Zeit begonnen, in der psychoanalytische Ausbildungsvereine offen lebenden Homosexuellen die Aufnahme zur Ausbildung verweigerten) geoutet haben (Drescher, 2008, S. 452). Jack Drescher zufolge waren diese Analytiker*innen meist Psycholog*innen, die nicht in Ausbildungsvereinen der APsaA (American Psychoanalytic Association) bzw. IPA (International Psychoanalytic Association) ausgebildet wurden und theoretisch stark von Strömungen ausserhalb des klassisch medizinisch-psychoanalytischen Diskurses beeinflusst waren, etwa von zunächst aus den Geisteswissenschaften hervorgegangenen Diskursen der Postmoderne und des Poststrukturalismus, von Gender Studies und Queer Theory. Seit Mitte der 2000er Jahre ist eine ähnliche Entwicklung in Bezug auf Trans* zu beobachten: Die ersten vereinzelten Trans*Personen wurden - zum Teil geoutet, zum Teil jedoch outeten sich die Personen erst im Laufe ihrer Ausbildung - als Kandidat*innen in US-amerikanischen psychoanalytischen Ausbildungsvereinen (die zumeist jedoch nicht Mitglied der IPA sind) zugelassen; zugleich ist auch eine progressivere Auseinandersetzung mit Trans*Themen in einzelnen Feldern der Psychoanalyse zu bemerken, die wesentlich durch die neu aufgenommenen queeren und trans* Analytiker*innen getragen und beeinflusst ist. Im Besonderen sei an dieser Stelle auf die Arbeiten von Jack Pula und Griffin Hansbury verwiesen (u. a. Hansbury, 2011; Pula, 2015). 
In den 70er und 80er Jahren kamen Kritiken an der Psychoanalyse und ihrem Umgang mit Homosexualität und nicht-konformen Geschlechtsidentitäten grossteils von ausserhalb des analytischen Feldes und aufgrund des pathologisierenden Diskurses innerhalb der Psychoanalyse nur zu einem kleinen Teil von Psychoanalytiker*innen selbst: Hier wurden zunächst vor allem alternative Lesarten des Ödipuskomplexes vorgeschlagen. Unter dem Einfluss postmoderner Theorien und durch die aktive Teilnahme von queeren Analytiker*innen hat sich der Fokus der Debatte schliesslich von der Ursachenforschung weg und hin zu einer Auseinandersetzung darüber verschoben, woraus sich die Homo- und Transphobie innerhalb der Psychoanalyse speist (Blechner, 1993; Drescher, 1995; Roughton, 2002; Lewes, 2005), wie sich Übertragungsbeziehungen zwischen queeren bzw. heterosexuellen Analytiker*innen in der Arbeit mit jeweils queeren bzw. heterosexuellen Patient*innen gestalten (Isay, 1991; Frommer, 1995, 1999; Hansbury, 2001; Burton und Gilmore, 2010) und auf welche Weise die klassischen psychoanalytischen Konzepte von Weiblichkeit und Männlichkeit zu dekonstruieren wären; die Breite und Differenz innerhalb der Homosexualität selbst wurde thematisiert und die Unterscheidung von Homo- und Heterosexualität infrage gestellt (Chodorow, 1992; O'Connor und Ryan, 1993; de Lauretis 1994; Drescher, 1997; Schwartz, 1998). Die Lebendigkeit der Debatte - vor allem in den USA - ist auch auf die Gründung des Journal of Gay and Lesbian Psychotherapy 1999 zurückzuführen, in dem nicht nur mehrheitlich schwule und lesbische Analytiker*innen publizieren, sondern dessen Editorial Board ebenfalls aus schwulen und lesbischen Analytiker*innen besteht (Drescher, 2008, S. 454). Daneben spielt die Fachzeitschrift Psychoanalytic Dialogues. A Journal of Relational Perspectives sowie überhaupt die relationale Schule eine wesentliche Rolle in der Debatte um entpathologisierende psychoanalytische Positionen zu Homo- und Transsexualität bis hin zu offen debattierten queeren Themen. Neben diesen beiden Zeitschriften sind insbesondere zwei Sammelbände hervorzuheben: Der von Thomas Domenici und Ronnie C. Lesser 1995 herausgegebene Sammelband mit dem Titel Disorienting Sexuality und der hier schon mehrfach zitierte von Tim Dean und Christopher Lane editierte Sammelband Homosexuality and Psychoanalysis von 2001. Die offene Auseinandersetzung mit entpathologisierenden und vor allem queeren Konzepten innerhalb der Psychoanalyse ist also recht jung.

\section{Queere Analytiker*innen - ein Überblick über einige Akteur*innen}

Im Folgenden möchte ich einige queere Analytiker*innen und deren Ansätze vorstellen. Ich beginne mit der amerikanischen Psychoanalytiker*in Betsy Kassoff, 
die zeigt, dass insbesondere die relationale Psychoanalyse von der Queer Theory beeinflusst wurde: "In classical analysis, core gender or gender identity, gender role, and sexual object choice are often conflated, or at the least expected to be congruent. One of the most powerful effects queer theory has had on relational psychoanalysis is what I call the queering of sexual identity stories" (Kassoff, 2004, S. 163). Kassoff hebt hervor, dass die Auseinandersetzung mit Queer Theory Analytiker*innen hilft, klassische Narrative von Weiblichkeit und Männlichkeit, aber auch Vorstellungen von Passivität und Aktivität kritisch zu hinterfragen, was für die Arbeit mit gender-queeren Analysand*innen notwendig ist (ebd., S. 165). Kassoff fragt darüber hinaus - Suzanne Iasenza (1995) folgend -, ob man Geschlecht nicht auch als primäres Organisationsprinzip von Begehren hinterfragen muss: "If gender is displaced as the primary organizer of sexual desire, other 'attractors' emerge, including '... gender role (masculinity and femininity), sexual orientation (gay, lesbian, bisexual), sexual agency (activity and passivity), power relations (dominance and submission, top and bottom), sexual act (oral or anal sex, paid sex, group sex, S/M, vaginal intercourse, bondage, leather, costumes), and other characteristics such as age, intimacy, body size, race, ethnicity, class, physical appearance and personality. In other words, some people may desire sexual acts or particular characteristics more than other people'” (Kassoff, 2004, S. 169). Zudem verweist sie auf die Bedeutung der Auseinandersetzung mit der Gegenübertragung in Bezug auf Geschlecht, sexuelle, dominante oder submissive Gefühle und Identifizierungen auf Seiten der Analytiker*innen. Kassoff greift Ken Corbetts Versuch auf, ein neues Entwicklungsmodell zu entwerfen und affirmiert vor allem seinen Ansatz, Entwicklung dynamisch zu denken: "a theory of process rather than state, of position rather than stage, of becoming rather than being" (Corbett, 2001, S. 322).

Corbett versteht die Kindheit eines Subjekts daher als "a resonant yet emergent process, open to oscillation and transformation within the multiple relational worlds constructed throughout a life" (ebd., S. 322). Es geht für ihn in der Folge daher nicht darum, danach zu fragen, warum jemand homosexuell ist, sondern wie jemand homosexuell ist, mit welchen Bedeutungen dies verbunden ist. In seinen zahlreichen Arbeiten zu homosexuellen Jungen arbeitet Corbett - ebenfalls Vertreter der relationalen Psychoanalyse - vielfältige Männlichkeitskonzepte heraus, die er in der klinischen Arbeit findet und die keineswegs lediglich Stereotypien reproduzieren (Corbett, 1996, 2009).

Der im vorliegenden Artikel ebenfalls bereits zitierte Psychiater* und Analytiker* Jack Drescher hat sich in seinen zahlreichen Publikationen immer 
wieder sowohl mit vor allem männlicher Homosexualität aber auch mit queeren Themen und Trans* auseinandergesetzt. Er hat u.a. Behandlungsmanuale für die therapeutische und analytische Behandlung von sowohl Homosexuellen als auch Transgenders verfasst (u. a. Drescher, 1998; Drescher et al., 2003; Drescher \& Byne, 2013) und ist ein vehementer Opponent von in den USA sehr verbreiteten Konversions-Therapien und der Ex-Gay Bewegung.

Die in Dublin lebende Analytiker*in Eve Watson geht in ihrem Artikel Queering Psychoanalysis / Psychoanalysing Queer explizit der möglichen produktiven Verbindung von Queer Theory und lacanianischer Psychoanalyse nach. "It seems to me that it is in relation to the twin fundamentals of Lacanian psychoanalysis, desire and subjectivity, that psychoanalysis and queer theory share a common purpose. For the Lacanian speaking subject is founded upon and motivated by desire which is originally independent of gender and thus it offers the basis for a radically non-normative and non-heterosexist account of sexuality and desire" (Watson, 2009, S. 117 f.). Tim Deans Lesart folgend hebt Watson hervor, dass Lacan einen für die Queer Theory radikalen Schritt vollzieht, indem er in seiner Begehrenskonzeption sex und gender voneinander trennt: "By theorizing sexuality in terms of the unconscious, Lacan divorces desire from anatomical constraints. His emphasis on language makes sexual desire an effect of representation rather than of biology" (Dean \& Lesser, 2001, S. 26). Sowohl Dean und Lane als auch Watson sehen in Lacans psychoanalytischem Ansatz auch einen identitätskritischen. "Lacan's conceptualisation of desire, which radically extends Freud's conceptualisation of sexuality, emerges independently of heterosexuality and homosexuality and therefore it dismantles the normalising implications of identity and object choice. This account of sexuality ought to be attractive to anti-normative politics" (Watson, 2009, S. 135). Watson sieht also wesentlich in Lacans Konzeption des Begehrens Potenzial für anti-normative Diskurse, da ihr zufolge die lacanianische Begehrenskonzeption Freuds Denken der Sexualität insofern überschreite, als sie unabhängig von der konkreten Begehrensausrichtung (Hetero- bzw. Homosexualität) gedacht werden kann. Dean und Lane betonen hingegen den identitätskritischen Charakter des Spiegelstadiums, da Lacan hier das Selbst bzw. die Identität über eine Täuschung hergestellt sieht (Lacan, 1991; Dean \& Lane, 2001, S. 7). ${ }^{5}$

Die in London arbeitende und lehrende Analytiker*in Anne Worthington arbeitet an der Schnittstelle von Psychoanalyse und Queer Theory vor allem ausgehend von Konzepten zu weiblicher Homosexualität. Worthington zeigt anhand von aktuellen Fallstudien zu homosexuellen Patient*innen, dass - obschon die 
Analytiker*innen sich der Genderproblematik bewusst sind und auf queere Ansätze zurückgreifen (etwa Gaier, 1995; Stack, 1995; Layton, 2004; u. a.) - die klinische Arbeit dennoch stark mit der Vorannahme einer männlichen Identifizierung arbeitet (Worthington, 2011).

Aus objektbeziehungstheoretischer Perspektive beschäftigt sich die ebenfalls in Dublin praktizierende Analytiker*in Noreen Giffney mit Queer Theory. Sie hat das Buch Queer Theory. The Key Concepts publiziert, in dem sie Queer Theory zusammen mit einer Reihe anderer Felder beleuchtet, u. a. ist für sie die Psychoanalyse ein wesentliches Schlüsselkonzept für queeres Denken (Giffney, 2010).

\section{Von den Akteur*innen zurück zum Feld}

In den letzten Jahren ist insbesondere die Auseinandersetzung mit Trans* innerhalb der Psychoanalyse zu einem aktuellen Thema geworden, das viele Fragen und neue Erkenntnisse darüber, was psychoanalytische Konzepte zu Sexualität, Identität, Körper usw. in Bezug auf Trans* leisten können, aufwirft. Im Rahmen der IPA-Konferenz in Boston 2015 gab es mehrere Panels zum Thema. Hier waren u. a. die schon erwähnten Analytiker* Pula und Hansbury unter den Vortragenden. Der Diskurs um Transgender bzw. Transsexualität oder -identität ist in der Psychoanalyse nach wie vor von stark pathologisierenden Tendenzen geprägt. Die Aufnahme von Transgenders als Kandidat*innen in analytische Ausbildungen wäre im deutschsprachigen Raum sehr zu begrüssen. Ob dies bereits eine denkbare Möglichkeit ist und in naher Zukunft auch Wirklichkeit werden könnte, ist derzeit noch zu bezweifeln.

«Gender Dysphoria» (DSM V) bzw. «Störung der Geschlechtsidentität» (ICD-10) stellt nach wie vor die entscheidende psychiatrische Diagnose dar, die für jene Transgenders, die medizinische Unterstützung in Anspruch nehmen, von Bedeutung ist, um in ihrem Wunsch den Körper zu verändern auch ernstgenommen zu werden und um diese Leistungen über Krankenkassen auch abrechnen zu können. Einerseits verhilft die genannte psychiatrische Diagnose Transgenders also zu einer gewissen Anerkennung ihres Wunsches, andererseits wird im gleichen Zug ein pathologisierender Diskurs fortgeschrieben. ${ }^{6}$ Insbesondere die Auseinandersetzung mit Trans* muss meiner Einschätzung nach auch in der Psychoanalyse zu einer neuen Auseinandersetzung mit Identität/en und Körper/n führen. Gerade die psychoanalytische Auseinandersetzung in der ihr eigenen Komplexität hätte das Potenzial, Pathologisierungen entgegenzuwirken und zu neuen Verstehens-Modi von Körper-Transformationen, Identifizierungen und Begehrensformen zu ver- 
helfen. In Assuming a Body arbeitet beispielsweise Gayle Salamon über eine verbindende Lesart von Phänomenologie, Psychoanalyse und Queer Theory eine Annäherung an Trans*-Körper heraus, ohne diese Körper jedoch zu fixieren oder ihre Spezifitäten endgültig zu definieren (Salamon, 2010). Psychoanalytisch bezieht sich Salamon wesentlich auf den Psychoanalytiker* und Zeitgenossen* Freuds Paul Schilder, der den Körper über ein Körper-Bild versteht, das nicht nur ein singuläres Bild darstellt, sondern eine Multiplizität von Bildern, die zeitlich veränderbar ist, über unsere Beziehungen zu anderen hergestellt wird und von den Konturen des Körpers abweicht (ebd., S. 29). Salamon weist mit Schilders Körper-Schema das auch in der Psychoanalyse gängige Verständnis zurück, dass lediglich Körper von Transgenders Produkt einer Fantasie wären, während Körper von genderkonformen Personen materieller, realer, wahrer und wirklicher wären (Salamon \& Corbett, 2011, S. 224).

Im deutschen Sprachraum ist die Auseinandersetzung mit Psychoanalyse und Queer Theory noch weitaus spärlicher gesät. Die Philosoph*in, Kultur- und Queer-Theoretiker*in Antke Engel hat sich immer wieder vor allem ausgehend von de Lauretis, Laplanche und Lacan mit psychoanalytischen Ansätzen beschäftigt. In Wider die Eindeutigkeit liest sie Butlers Konzept des «lesbischen Phallus» kritisch, da die lacansche Position des «Phallus-Habens» von Butler nicht dekonstruiert wird. Dagegen schliesst sich Engel der Position von de Lauretis an, die mit ihrer Konzeption des Fetischs die Signifikanten vervielfältigen würde (Engel, 2002, S. 169 ff.). «Fetische sind Zeichen oder Objekte des Begehrens, die die symbolische Rückgewinnung eines verlorenen Frauenkörpers inszenieren und damit eine weibliche Körper-Imago fundieren, die narzisstisch und libidinös besetzt werden kann» (ebd., S. 173). Engel versteht den Phallus der Konzeption von Teresa de Lauretis gemäss als Fetisch; wobei umgekehrt ein Fetisch nicht der Phallus ist. Bersani zufolge muss das Begehren mit Engel nicht an den privilegierten Phallus gebunden sein, jedes Objekt kann Objekt des Begehrens werden, indem es «bestimmte Phantasieszenarien aufruft und aufführt» (ebd., S. 174). Schliesslich gilt es an dieser Stelle auf die Arbeiten der Psychoanalytiker*in Ilka Quindeau hinzuweisen, die in ihren Texten immer wieder die grundlegende Unterscheidung von Homo- und Heterosexualität in Frage stellt (Quindeau, 2014, S. 99) und vorschlägt, Geschlechtsidentität nicht dichotom sondern in Form eines Kontinuums zu denken (ebd., S. 84).

In Wien hat sich 2014 das Forschungskollektiv Queering Psychoanalysis gegründet, in dem ich gemeinsam mit meinen Kolleg*innen Barbara Zach und 
Anke Müller Morocutti an einem Feld arbeite, in dem Psychoanalyse und Queer Theory als füreinander produktive Theorien wirksam werden. ${ }^{7}$

\section{Queer Theory und Psychoanalyse: Ein produktives Verhältnis}

Seit 1987 ist Homosexualität - bis dahin als psychische Erkrankung eingestuft - aus dem Diagnose-Manual (DSM) der American Psychiatric Association und schliesslich 1992 aus dem ICD (International Statistical Classification of Diseases and Related Health Problems) derWHO gestrichen worden, und mittlerweile nehmen auch psychoanalytische Ausbildungsvereine homosexuelle Kandidat*innen auf (was transgender bzw. transidente Personen anbelangt, ist der Diskurs von einer Entpathologisierung noch weiter entfernt). Der stereotype und diskriminierende Diskurs in der Psychoanalyse hat sich jedoch kaum verändert, obzwar Schwulen und Lesben - in vielfacher Hinsicht - nicht mehr offen homophob begegnet wird.

Queer Theory stellt eine Zugangsweise dar, Normen und Normierungsprozesse nachhaltig zu hinterfragen, zu dekonstruieren und entpathologisierende Zugänge zu sexuellen, geschlechtlichen, identifikatorischen usw. Phänomenen zu gewinnen. Queer Theory ist damit alles andere als weit von den Ansätzen der Psychoanalyse entfernt: "Psychoanalytic theory helps us to recognize and begin to identify the multiple functions of a patient's experiences in treatment. In its appreciation for the fluidity of gender and sexual identities, queer theory can help us to resist temptations to label and, instead, to retain an open mind. Both theories can help us to live with uncertainty without abandoning what we know. [...] Queer theory invites us to think in a more complex manner about sexuality, gender, identity, and desire. Like psychoanalysis, it is in a state of motion; it is continually changing, expanding, and being revised by its diverse constituency." (Grossman, 2002, 296 f.) Begehren, Identitäten und deren jeweilige Genealogien gerade nicht als Prozesse der Schliessung zu betrachten, die allzu einfach zu definieren sind, sondern sie als Prozesse des Werdens, des Kontinuums und der Transformation ernst zu nehmen und dieser Herausforderung ethisch zu begegnen, dazu liefern uns Psychoanalyse und Queer Theory gemeinsam produktives Denkmaterial.

\section{Literatur}

Bersani, L. (1987). Is the Rectum a Grave? AIDS. Cultural Analysis/Cultural Activism $43,197-222$.

Bersani, L. (1995). Homos. Cambridge/Massachusetts: Harvard University Press. Blechner, M. J. (1993). Homophobia in psychoanalytic writing and practice. Psychoanalytic Dialogues 3, 627-637. 
Burton, J. K. \& Gilmore, K. (2010). “This Strange Disease”. Adolescent Transference and the Analyst's Sexual Orientation. Journal of the American Psychoanalytic Association 58, 715-734.

Butler, J. (1991). Das Unbehagen der Geschlechter. Frankfurt a. M.: Suhrkamp.

Butler, J. (1997). Körper von Gewicht. Die diskursiven Grenzen des Geschlechts. Frankfurt a. M.: Suhrkamp.

Butler, J. (2001). Psyche der Macht. Das Subjekt der Unterwerfung. Frankfurt a. M.: Suhrkamp.

Chodorow, N. J. (1992). Heterosexuality as a compromise formation: Reflections on the Psychoanalytic Theory of Sexual Development. Psychoanalysis and Contemporary Thought 15, 267-304.

Corbett, K. (1996). Homosexual Boyhood. Gender and Psychoanalysis 1, 429-461.

Corbett, K. (2001). More Life. Psychoanalytic Dialogues 11, 313-335.

Corbett, K. (2009). Boyhoods: Rethinking masculinities. New Haven: Yale University Press.

Dean, T. (2000). Beyond sexuality. Chicago: University of Chicago Press.

Dean, T. \& Lane, C. (2001). Homosexuality and Psychoanalysis. An Introduction. In: Dies. (Hrsg.) Homosexuality and psychoanalysis. Chicago: University of Chicago Press. S. 3-42.

de Lauretis, T. (1994). The practice of love. Lesbian sexuality and perverse desire. Bloomington: Indiana University Press.

Drescher, J. (1995). Anti-Homosexual Bias in Training. In: T. Domenici \& R. Lesser (Hrsg.), Disorienting psychoanalysis. Psychoanalytic reappraisals of sexual identities, New York: Routledge, S. 227-241.

Drescher, J. (1997). From preoedipal to postmodern: Changing psychoanalytic attitudes toward homosexuality. Gender \& Psychoanalysis 2 (2), S. 203-216.

Drescher, J. (1998). Psychoanalytic therapy and the gay man. New York: Routledge.

Drescher, J., D’Ercole, A. \& Schoenberg, E. (Hrsg.) (2003). Psychotherapy with gay men and lesbians. Contemporary dynamic approaches. NewYork: Routledge.

Drescher, J. (2007). From bisexuality to intersexuality. Rethinking gender categories. Contemporary Psychoanalysis 43 (1), 204-228.

Drescher, J. (2008). A History of Homosexuality and Organized Psychoanalysis. Journal of The American Academy of Psychoanalysis and Dynamic Psychiatry 36 (3), 443-460.

Drescher, J. \& Byne, W. (Hrsg.) (2013). Treating transgender children and adolescents. An interdisciplinary discussion. New York: Routledge. 
Domenici, T. \& Lesser, R. C. (Hrsg.) (1995). Disorienting sexuality. Psychoanalytic reappraisals of sexual identities. New York: Routledge.

Engel, A. (2002). Wider die Eindeutigkeit. Sexualität und Geschlecht im Focus queerer Politik und Repräsentation. Frankfurt a. M.: Campus Verlag.

Foucault, M. (1983). Der Wille zum Wissen. Sexualität und Wahrheit 1. Frankfurt a. M.: Suhrkamp.

Freud, S. (1905): Drei Abhandlungen zur Sexualtheorie I. Die sexuellen Abirrungen. Gesammelte Werke: V, S. 33-72.

Freud, S. (1923). Das Ich und das Es. Gesammelte Werke: XIII, S. 237-289.

Frommer, M. S. (1995). Countertransference Obscurity in the Psychoanalytic Treatment of Homosexual Patients. In: T. Domenici \& R. Lesser (Hrsg.): Disorienting psychoanalysis. Psychoanalytic reappraisals of sexual identities. New York: Routledge, S. 65-82.

Frommer, M. S. (1999). Reflections on self-disclosure, desire, shame and emotional engagement in the gay male psychoanalytic dyad. Journal of Gay and Lesbian Psychotherapy 3 (1), 53-65.

Gair, S. R. (1995). The False Self, Shame and Challenge of Self Cohesion. In: J. M. Glassgold \& S. Iasenza (Hrsg.): Lesbians and psychoanalysis: Revolutions in theory and practice. New York \& London: Free Press, S. 107-123.

Giffney, N. (2010). Queer Theory. The key concepts. Oxford: Berg Publishers.

Grossman, G. (2002). Queering Psychoanalysis. The Annual of Psychoanalysis 30, 287-299.

Hansbury, G. (2011). King Kong \& Goldilocks: Imagining Transmasculinities Through the Trans-Trans Dyad. Psychoanalytic Dialogues 21, 210-220.

Hocquenghem, G. (1974). Das homosexuelle Verlangen. München: Carl Hanser Verlag.

Hutfless, E. (2011). In Dir mehr als Dich. Phänomenologien des Begehrens zwischen diskursiver Produktion und leiblichem Zur-Welt-Sein. Würzburg: Königshausen \& Neumann.

Iasenza, S. (1995). Platonic pleasures and dangerous desires: Psychoanalytic theory, sex research, and Lesbian sexuality. In: J. M. Glassgold \& S. Iasenza (Hrsg.), Lesbians and psychoanalysis: Revolutions in theory and practice. New York: Free Press, S. 345-373.

Isay, R. A. (1991). The Homosexual Analyst-Clinical Considerations. Psychoanalytic Study of the Child 46, 199-216.

Kassoff, B. (2004). The Queering of Relational Psychoanalysis. Journal of Lesbian Studies 8 (1-2), 159-176. 
Lacan, J. (1991). Das Spiegelstadium als Bildner der Ichfunktion, wie sie uns in der psychoanalytischen Erfahrung erscheint. In: J. Lacan: Schriften I. Bd. 1. Weinheim/Berlin: Quadriga, S. 61-70.

Layton, L. (2004). Who's that girl? Who's that boy? Clinical practice meets post-modern gender theory. New York: The Analytic Press.

Lewes, K. (2005). Homosexuality, Homophobia, and Gay-Friendly Psychoanalysis. Fort Da 11, 13-34.

Pula, J. (2015). Understanding Gender Through the Lens of Transgender Experience. Journal of Psychoanalytic Inquiry 35 (8), 809-822.

Richardson, D. \& Seidman, S. (Hrsg.) (2002). Handbook of lesbian and gay studies. London: Sage Publications.

Roughton, R. E. (2002). Rethinking Homosexuality: What it Teaches us about Psychoanalysis. Journal of the American Psychoanalytic Association 50, 733-763.

O’Connor, N. \& Ryan, J. (1993). Wild desires and mistaken identities. Lesbianism. New York: Columbia University Press.

Salamon, G. (2010). Assuming a body. Transgender and rhetorics of materiality. New York: Columbia University Press.

Salamon, G. \& Corbett, K. (2011). Speaking the Body/Mind Juncture: An Interview With Gayle Salamon on Assuming a Body: Transgender and Rhetorics of Materiality. Psychoanalytic Dialogues 21, 221-229.

Schwartz, A. E. (1998). Sexual subjects: Lesbians, gender and psychoanalysis. New York: Routledge.

Stack, C. (1995). The Lesbian Patient: Narratives of Subjectivity, Gender and Sexual Identity in Lesbians and Psychoanalysis. In: J. M. Glassgold \& S. Iasenza, S. (Hrsg.): Lesbians and psychoanalysis: Revolutions in theory and practice. New York \& London: Free Press, S. 327-344.

Quindeau, I. (2014). Sexualität. Giessen: Psychosozial-Verlag.

Watson, E. (2009). Queering Psychoanalysis/Psychoanalysing Queer. Annual Review of Critical Psychology 7, 114-139.

Worthington, A. (2011). Female homosexuality: Psychoanalysis and Queer Theory. Online: https://eprints.mdx.ac.uk/7222/1/Worthington_PhD.pdf; Siehe auch: https://www.inter-disciplinary.net/wp-content/uploads/2011/04/ worthingtonqpaper.pdf [27.12.2015]. 


\section{Anmerkungen}

1 Im vorliegenden Text verwende ich den Asteriscus $\left(^{*}\right)$ als Markierung einer Leerstelle zwischen den sprachlich und gesellschaftlich konformen Polen männlich und weiblich, um Raum für vielfältige sexuelle und geschlechtliche Lebens- und Seinsweisen jenseits des heteronormativen Dualismus Mann/Frau, männlich/weiblich zu eröffnen und den Ausschluss dieser im konventionellen Sprachgebrauch sichtbar zu machen.

2 Es sei an dieser Stelle darauf verwiesen, dass «Queer Theory» keine einheitliche oder homogene Theorie darstellt, sondern vielmehr ein plurales Feld der Auseinandersetzungen markiert.

3 LGBTIQ* steht hier für LesbianGayBiTransInterQueer und wird durch den Asteriscus noch für weitere Bedeutungen geöffnet.

$4 \quad$ Die Kritik an der Mangelkonzeption des Begehens in der Psychoanalyse wurde in den 70er Jahren bereits von feministischen Theoretiker*innen wie Luce Irigaray und Hélène Cixous vorgebracht.

$5 \quad$ Im theoretischen Ansatz Lacans ist das Kind mit einem anatomisch nicht ausgereiften Körper ausgestattet, der sich seiner Kontrolle entzieht und der zunächst als mangelhaft und fragmentiert erlebt wird. Im Spiegel erblickt das Kind sein Ebenbild, es erkennt und verkennt sich zugleich als ganzheitliche Oberfläche. Mithilfe des Spiegelbildes und durch Bestätigung von aussen entsteht ein imaginäres Körper-Bild, mithilfe dessen der ursprüngliche Mangel für das kleine Subjekt «beseitigt» werden kann. Die spiegelbildliche Identifizierung ist einerseits mit Lacan nie eine vollständige - es bleibt ein Rest, eine Entfremdung zwischen realem Körper und imaginärem Abbild und sie ist andererseits immer eine Täuschung (Lacan, 1991; Hutfless, 2011, S. 26f.). Diesen Ansatz findet man nicht erst bei Lacan; Freud schreibt in «Das Ich und das Es»: «Das Ich ist vor allem ein körperliches, es ist nicht nur ein Oberflächenwesen, sondern selbst die Projektion einer Oberfläche» (Freud, 1923, S. 253).

6 Seit einigen Jahren gibt es immer wieder Initiativen, die sich für die Streichung von «Geschlechtsidentitätsstörungen» aus den Diagnosemanualen einsetzen und die Behandlung von Trans* als psychiatrische Störung kritisieren. Siehe dazu etwa: http://www.stp2012. info/old/de [28.12.2015] oder http://ansaraonline.com/yahoo_site_admin/assets/docs/ APA_Response_Letter_2012_Updated_version_10th_September_Ansara_Friedman_Blumer_ Fryer_Nic_Giolla_Easpaig_Richmond_Samons.253222842.pdf [28.12.2015].

7 Siehe dazu den Blog: https:/ queeringpsychoanalysis.wordpress.com/ [28.12.2015]. Aktuell ist ein Sammelband in Arbeit, der 2017 im Verlag Zaglossus erscheinen wird.

\section{Angaben zur Autorin}

Esther Hutfless, Mag.a Dr.in, 1980, arbeitet als Philosoph*in und Psychoanalytiker*in in Wien. Ihre* Forschungsschwerpunkte liegen in den Bereichen Phänomenologie, Psychoanalyse, Dekonstruktion, Poststrukturalismus, Gender Studies und Queer Theory. Sie* ist eine der Gründer*innen der Forschungsgruppe Queering Psychoanalysis. 